SUBJECT AREAS: OPTICAL PROPERTIES AND DEVICES

APPLIED PHYSICS

Received 30 January 2014

Accepted

20 June 2014

Published

7 July 2014

Correspondence and requests for materials should be addressed to S.-H.C. (sukho@khu. ac.kr)

* These authors contributed equally to this work. \section{graphene-quantum-dot photodetectors}

\author{
Chang Oh Kim ${ }^{1 *}$, Sung Won Hwang ${ }^{2 *}$, Sung Kim*, Dong Hee Shin', Soo Seok Kang', Jong Min Kim', \\ Chan Wook Jang', Ju Hwan Kim' ', Kyeong Won Lee', Suk-Ho Choi' \& Euyheon Hwang ${ }^{3}$
}

'Department of Applied Physics and Institute of Natural Sciences, College of Applied Science, Kyung Hee University, Yongin $446-$ 701, Korea, ${ }^{2}$ Advanced Development Department, Samsung Electronics Co., Ltd, Yongin 446-71 1, Korea, ${ }^{3}$ SKKU Advanced Institute of Nanotechnology, Sungkyunkwan University, Suwon 440-746, Korea.

Graphene quantum dots (GQDs) have received much attention due to their novel phenomena of charge transport and light absorption/emission. The optical transitions are known to be available up to $\sim 6 \mathrm{eV}$ in GQDs, especially useful for ultraviolet (UV) photodetectors (PDs). Thus, the demonstration of photodetection gain with GQDs would be the basis for a plenty of applications not only as a single-function device in detecting optical signals but also a key component in the optoelectronic integrated circuits. Here, we firstly report high-efficient photocurrent (PC) behaviors of PDs consisting of multiple-layer GQDs sandwiched between graphene sheets. High detectivity $\left(>10^{11} \mathrm{~cm} \mathrm{~Hz}^{1 / 2} / \mathrm{W}\right)$ and responsivity $(0.2 \sim 0.5$ $\mathrm{A} / \mathrm{W}$ ) are achieved in the broad spectral range from UV to near infrared. The observed unique PD characteristics prove to be dominated by the tunneling of charge carriers through the energy states in GQDs, based on bias-dependent variations of the band profiles, resulting in novel dark current and PC behaviors.

thas been demonstrated that graphene can be cut in well-confined nanometer-sized pieces, such as graphene nanoribbons ${ }^{1,2}$ and graphene quantum dots $(\mathrm{GQDs})^{3-5}$, which provide the opportunity to explore novel structural $^{6}$, optical ${ }^{4,5}$, and electrical phenomena ${ }^{7}$ not obtainable in other materials. Graphene is a single atomic layer of graphite with an infinite exciton Bohr radius due to its linear energy dispersion relation of the charge carriers $^{8,9}$, resulting in quantum confinement effect for graphene of any finite size $\mathrm{e}^{7,10}$. It has been reported that three different photon energy scales are involved in the optical transitions of GQDs ${ }^{11} ; \approx 2.8 \mathrm{eV}$ at full bandgap, $\approx 1.4 \mathrm{eV}$ at half bandgap, and terahertz energies, which can make GQDs a particularly promising material for high gain photodetection in a broad wavelength range. Blue-luminescent GQDs in water have been shown to exhibit absorption spectra peaked at energies up to $\sim 6 \mathrm{eV}^{4,12}$, especially useful for ultraviolet (UV) photodetectors (PDs).

PDs in optoelectronic integrated circuits (OEICs) usually have p-n or p-i-n junction diode structures or bipolar transistor structures fabricated using standard complementary metal oxide semiconductor (CMOS) technology ${ }^{13,14}$, which requires many device-processing steps, thereby increasing the fabrication complexity and cost. The development of a simple PD structure is therefore highly desired not only for single-function PDs, but also for integrated PDs. Si is a good material as PDs for the visible and near infrared (VIS-NIR) wavelength range, but not useful for UV detection. Wide-bandgap compound semiconductors are currently used for UV detection ${ }^{15}$, but require fabrication processes not compatible with Si technology. Compound-semiconductor quantum-dot PDs have recently emerged as top-surface detectors offering integration with circuit technologies due to the low cost and high sensitivity/tunability in the VIS and infrared ${ }^{16}$ regions, but their performance is limited by the relatively poor carrier mobility $\left(10^{-3}-1 \mathrm{~cm}^{2} / \mathrm{Vs}\right)^{17}$. Graphene, on the contrary, exhibits very high mobility reaching $\sim 60,000 \mathrm{~cm}^{2} / \mathrm{Vs}$ on a BN substrate, and is a very thin, flexible, and durable material, suitable for transparent and foldable devices.

Previous studies have shown that photocurrent (PC) is generated in a graphene sheet near metallic contacts ${ }^{18}$ or at a graphene single-/bi-layer interface ${ }^{19}$. The $\mathrm{PC}$ response was attributed to the presence of potential steps that act as transport barriers at the metal contacts and could be modulated by sweeping gate voltages ${ }^{20-22}$. On the other hand, atomically thin heterostructures of two-dimensional semiconductors/graphene have presented strong light-matter interactions that can lead to large photon absorption and PC production ${ }^{23}$. However, from all these studies, the magnitudes of PCs in graphene sheet were shown to be typically very weak compared to those in conventional semiconductor PDs. It is clear that if GQDs can be used as a PD material in a broad wavelength range including UV, it will not only significantly increase the capability of the current Si VIS-NIR PDs, but also 


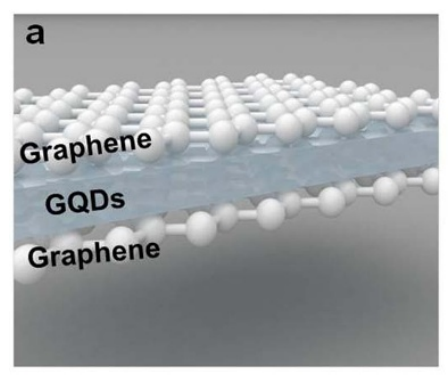

b

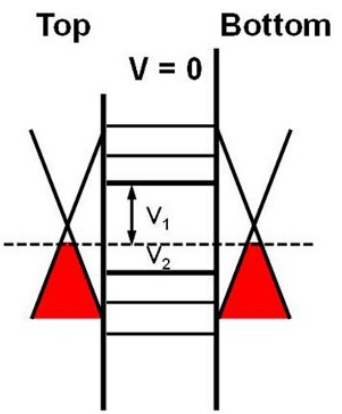

C
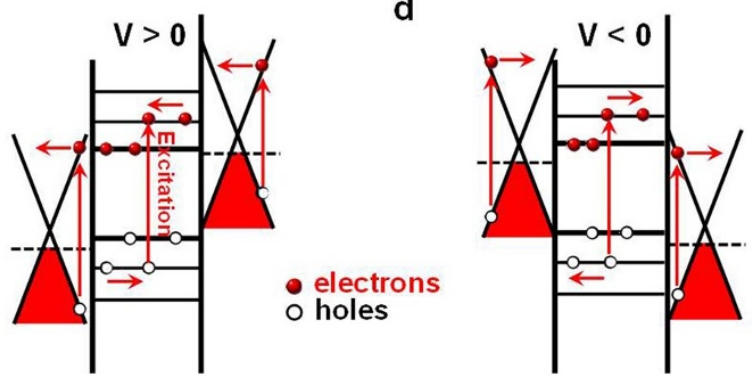

Figure $1 \mid$ Diagrams of schematics and band structure describing the PDs. Schematics of (a) a typical GQD PD device and its band diagrams under (b) no, (c) forward (positive), and (d) reverse (negative) biases. Red and blank spots represent electrons and holes, respectively, and lateral arrows indicate the transport directions of electrons and holes contributing to dark- and photo-currents. Here, $\mathrm{eV}_{1}\left(\right.$ or $\mathrm{eV}_{2}$ ) represents the energy between the Fermi energy of bottom graphene and the lowest unoccupied molecular orbital (or between the Fermi energy of top graphene and the highest occupied molecular orbital) of the GQD.

add new functionalities to the OEICs. Here, we report a very simple GQD-based structure that works as a PD showing strong PC responsivity in the UV-VIS-NIR range. The structure is based on multiple layers of GQDs sandwiched between single-layer graphene sheets. The PD structures permit large PC flow by tunneling of charge carriers through the energy states in GQDs.

\section{Results}

Dark and photo I-V characteristics. Figure 1a shows a schematic diagram of a typical graphene/GQDs/graphene PD (see Supplementary Fig. S1 for the preparation details), composed of slightly-p-type single-layer graphene sheets (Supplementary Figs. S2 and S3) and multiple layers of GQDs with a total thickness of $\sim 45 \mathrm{~nm}$, randomly distributed between the graphene sheets (Supplementary Fig. S4). The GQDs used in this sandwich device are estimated to be $\sim 17 \mathrm{~nm}$ in average size and produce a photoluminescence (PL) spectrum similar to that of GQDs in DI water, as characterized in our previous work ${ }^{12}$, whilst, a control sample, the graphene/graphene junction without GQDs, shows no PL (Supplementary Fig. S5a). These results demonstrate that the GQDs well exist in the sandwich structure and almost no structural alterations of GQDs occur during the GQD PD fabrication.

Positive voltages were applied to the Ag electrode on upper graphene layer with respect to the Ag electrode on lower graphene layer/ $\mathrm{SiO}_{2}$ under forward bias (Supplementary Fig. S6a). The dark current (DC)-voltage (I-V) curve of the graphene/graphene control sample is symmetric and linear in the forward/reverse directions with respect to zero voltage, indicating no tunneling-diode behavior (Supplementary Fig. S5b). The I-V curve of the control sample is almost not varied even under illumination at various photon wavelengths from 300 to $1000 \mathrm{~nm}$, resulting in no PC (Supplementary Fig. S6b).

In contrast, the dark I-V curve of the GQD device exhibits asymmetric and nonlinear properties with varying bias voltage, as shown
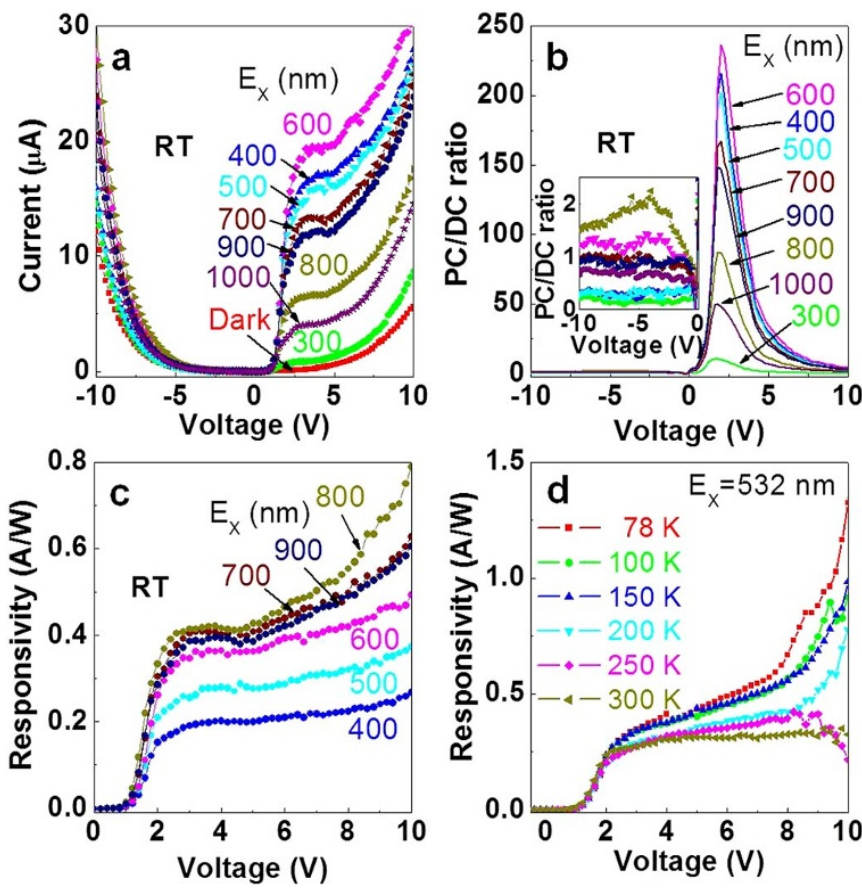

Figure $2 \mid$ Dark and photo I-V characteristics and bias-dependent responsivity. (a) I-V characteristics under dark and illumination at various photon wavelengths from 300 to $1000 \mathrm{~nm}$. (b) On/off current ratios as functions of bias voltage at various photon wavelengths from 300 to $1000 \mathrm{~nm}$. The inset shows a magnified version in the reverse bias (c) Responsivity as functions of forward bias at various photon wavelengths from 400 to $900 \mathrm{~nm}$. (d) Responsivity as functions of forward bias at various measurement temperatures from 78 to $300 \mathrm{~K}$.

in Fig. 2a (and Supplementary Fig. S5b), typical in tunneling diodes. The forward-biased DC is larger than the reverse-biased DC below $\sim 7.2 \mathrm{~V}$, but vice versa above $\sim 7.2 \mathrm{~V}$. These I-V characteristics can be attributed to tunneling through the available density of states (DOS) of GQDs between the metallic graphene sheets and well explained based on the band structures of biased graphene/GQDs/ graphene devices, as shown in Figs. 1c and d. We believe both graphene sheets and the GQDs are slightly p-type, as remarked above. When a forward bias $(\mathrm{V}>0)$ is applied a current will flow if the bias voltage exceeds $V_{1}$, where $\mathrm{eV}_{1}$ is the energy between the Fermi energy of bottom graphene and the lowest unoccupied molecular orbital of the GQD. For a negative bias $(\mathrm{V}<0)$ the current starts to flow for $\mathrm{V}$ $<-V_{2}$, where $\mathrm{eV}_{2}$ is the energy between the Fermi energy of top graphene and the highest occupied molecular orbital of the GQD.

The transport of electrons at the GQD layer may be induced by the thermionic emission above the barrier and the direct tunneling through the barrier. However, at low temperatures, we expect that the thermionic emission is very small and tunneling processes can dominate the transport. As an indication of the tunneling behavior ${ }^{24}$, the local minimum of current arises at a point minimizing the available DOS when the measurement temperature $(\mathrm{T})$ is lowered below $\sim 200 \mathrm{~K}$ (Supplementary Fig. S7), possibly resulting from the reduced thermionic emission at lower $\mathrm{T}$. The thermionic emission starts to be dominant above a threshold voltage that depends on $\mathrm{T}$ $(\sim 2.7 \mathrm{~V}$ at $78 \mathrm{~K}, \sim 1.7 \mathrm{~V}$ at $100 \mathrm{~K}, \sim 1.4 \mathrm{~V}$ at $150 \mathrm{~K}$, and no tunneling behaviors are observed at temperatures $\geq 200 \mathrm{~K})$. Such current minimum was not observed in the control sample (Supplementary Fig. S8). Since the tunneling process is seen at $T \leq 150 \mathrm{~K}$, we estimate that the potential barrier between bottom graphene and GQD is $\sim 2.2 \mathrm{~V}$ and that the barrier between top graphene and GQD is $\sim 1.4 \mathrm{~V}$ (Supplementary Fig. S7), resulting in the asymmetric DC $\mathrm{I}-\mathrm{V}$ characteristics of the device (Fig. 2a and Supplementary 
Fig. S5b). There have been several publications ${ }^{25,26}$ demonstrating the doping or charging effect of graphene at the graphene $/ \mathrm{SiO}_{2}$ interface. Therefore, the difference of the potential barriers may arise from the trapped charges between $\mathrm{SiO}_{2}$ substrate and bottom graphene which act as an effective potential. The barrier difference is reflected in the conduction band of the GQD of the device, as shown in Figs. 1b-d. Thus, for the voltages lower than the barriers the low-T DC is dominated by the quantum mechanical tunneling of carriers through the potential barrier. However, at high $\mathrm{T}$ or for photoexcited hot carriers the thermionic emission process is the main mechanism for the current, resulting in a sharp increase of DC for voltages above the barrier heights (Supplementary Fig. S7).

Fig. 2a also shows the PC of the device. For $\mathrm{V}>0$ the measured total current $(=\mathrm{DC}+\mathrm{PC})$ is much larger than $\mathrm{DC}$ at the same voltage, and PC exceeds DC by at least an order of magnitude. However, the total current for $\mathrm{V}<0$ increases slightly from DC, and PC is at most a factor of two times larger than DC at the same voltage. Due to the multiple scattering of light between GQDs and top graphene most absorption of light takes place in the GQDs. Absorption of photons in the GQDs produces electron-hole pairs. For $\mathrm{V}>0$ both electrons and holes photoexcited in the GQDs contribute to the total current, as described in Fig. 1c. However, due to the lower potential barrier between GQDs and top graphene the photoexcited electrons contribute mostly to PC. In addition to the current from photoexcited electrons and holes, the carrier multiplication by impact ionization also contributes to the enhancement of the total current. In graphene, since the relaxation due to the carrier-phonon scattering is much slower than that due to the carrier-carrier scattering ${ }^{27,28}$ a single photon produces multiple electronhole excitations by impact ionization induced by a hot electron ${ }^{29-31}$. For $\mathrm{V}<0$ photoexcited electrons in the GQD transport to bottom graphene, as shown in Fig. 1d. The higher potential barrier between GQDs and bottom graphene reduce PC. In this process, the current from photoexcited holes is limited due to the higher potential barrier between GQDs and top graphene for holes. Thus, for the reverse bias the total current is not almost affected by the photon absorption. The observed slight enhancement of the total current under illumination can be understood from the carrier multiplication. At high voltages the total I-V curves become more symmetric, almost irrespective of incident light wavelength, as shown in Fig. 2a, because the kinetic energy of carriers exceed the potential barriers on both sides and hence, the tunneling current is not affected by the barriers. These $\mathrm{I}-\mathrm{V}$ characteristics under dark as well as under illumination were almost not varied by using Au as electrode (Supplementary Fig. S9).

Photosensitivity and detectivity. The photoresponse strongly depends on the photon wavelength. The ratio of PC to DC or the on/off ratio shows maximum intensities at bias voltages of around 1.6 $\sim 2.1 \mathrm{~V}$ for various wavelengths $(\lambda)$, as shown in Fig. $2 \mathrm{~b}$. At lower T, the PC to DC ratio is strongly enhanced due to the sharp reduction of DC (Supplementary Figs. S10 and S11). Fig. 2c shows responsivity (the electrical current response to the incident optical power, $\mathrm{R}_{\mathrm{i}}$ ), measured as a function of bias voltage for various $\lambda$. There are no big variations in $\mathrm{R}_{\mathrm{i}}$ from $\sim 2.5$ to $\sim 5.5 \mathrm{~V}$, irrespective of $\lambda . \mathrm{R}_{\mathrm{i}}$ at $\lambda \sim$ $800 \mathrm{~nm}$ reaches $\sim 0.5 \mathrm{AW}^{-1}$ at $5.5 \mathrm{~V}$. At $400 \mathrm{~nm}, \mathrm{R}_{\mathrm{i}}$ is reduced to $\sim 0.2 \mathrm{AW}^{-1}$ because less light was absorbed by the device. These $\mathrm{R}_{\mathrm{i}}$ values are comparable to those reported for commercial $\mathrm{Si}$ and InGaAs $\mathrm{PDs}(\sim 0.5 \text { and } \sim 0.9 \mathrm{~A} / \mathrm{W}(4.2 \mathrm{~K}) \text {, respectively })^{32,33}$. Considerable values of $\mathrm{R}_{\mathrm{i}}$ are measured in certain ranges of $\lambda$ even for $\mathrm{V}<0$ due to the large absolute values of PC (Supplementary Fig. $S 12$ ). Fig. $2 d$ shows $R_{i}$ as a function of bias voltage at various $T$ 's when the PC is excited at $\lambda=532 \mathrm{~nm}$. The $R_{i}$ shows a big increase at $T \leq$ $200 \mathrm{~K}$, especially when the bias voltage increases above $\sim 8 \mathrm{~V}$. The DC gradually increases with increasing bias voltage at each temperature and converges to almost an identical value at $10 \mathrm{~V}$, irrespective of temperature (Supplementary Fig. S7a). In contrast,

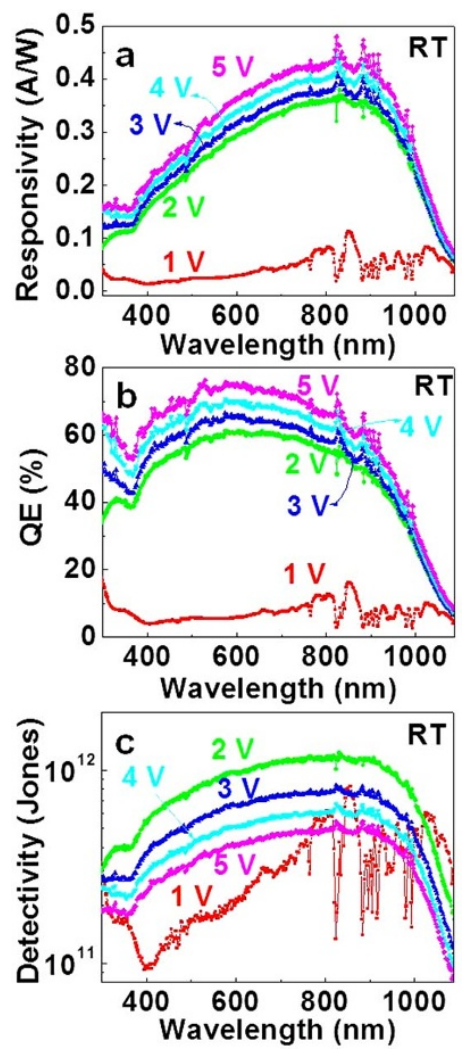

Figure 3 Spectral sensitivity and detectivity. (a) Spectral responsivities at different forward-bias voltages from 1 to 5 V. (b) Spectral quantum efficiencies at different forward-bias voltages from 1 to 5 V. (c) Spectral detectivities at different forward-bias voltages from 1 to $5 \mathrm{~V}$.

the total current $(\mathrm{DC}+\mathrm{PC})$ under illumination almost saturates up to $\sim 8 \mathrm{~V}$, but above $\sim 8 \mathrm{~V}$ it increases more rapidly than the DC does (Supplementary Fig. S10), resulting in such a big increase of $\mathrm{R}_{\mathrm{i}}$ (proportional to $\mathrm{PC}$ ) above $8 \mathrm{~V}$ at low temperatures. Higher bias voltage will enhance the collection of the photoexcited carriers, thereby increasing PC.

Fig. $3 a$ shows the spectral $R_{i}$ for voltages from 1 to $5 \mathrm{~V}$. There are small variations in the spectral $R_{i}$ above $2 \mathrm{~V}$. The quantum efficiency (QE) also gradually increases with increasing the bias above $2 \mathrm{~V}$ and is almost spectrally uniform with no clear peak in the visible range from 400 to $800 \mathrm{~nm}$, as shown in Fig. 3b. By considering the spectral $R_{i}$ in Fig. 3a, it is noted that a strong enhancement is especially reflected in high-energy side of the QE, consistent with the fact that the absorption of the GQDs is strongly enhanced at high energies ${ }^{4,12}$. The QE reaches around $80 \%$ in the visible range under a bias of $5 \mathrm{~V}$, which will be explained later in detail based on the transient PC data.

A key figure of merit of a PD is the noise equivalent power (NEP). This parameter is defined as the amount of incident light power that generates a PC equal to the noise current. NEP can be expressed as: [ref. 34] NEP $=\left(\left\langle I_{n}{ }^{2}\right\rangle\right)^{1 / 2} / \mathrm{R}_{\mathrm{i}}$ where $\left\langle I_{n}{ }^{2}\right\rangle$ is the time-averaged square of the total noise current and can be estimated by integrating spectral density of noise power (Supplementary Fig. S13a). Then, NEP can be calculated by using the spectral $\mathrm{R}_{\mathrm{i}}$ data in Fig. 3a (Supplementary Fig. $\mathrm{S} 13 \mathrm{~b})$. Another parameter used to characterize the performance of PDs is the detectivity $\left(D^{*}\right)$, which is related to NEP by the expression: $\mathrm{D}^{*}=(\mathrm{A} \Delta \mathrm{f})^{1 / 2} / \mathrm{NEP}$. Here, $\mathrm{A}$ is the effective area $\left(\sim 0.5 \times 0.5 \mathrm{~cm}^{2}\right)$ of the detector in $\mathrm{cm}^{2}$ and $\Delta \mathrm{f}$ is the electrical bandwidth in $\mathrm{Hz}$. With increasing the bias above $2 \mathrm{~V}$, the $\mathrm{D}^{*}$ decreases over almost full range of $\lambda$, as shown in Fig. $3 \mathrm{c}$. The maximum $\mathrm{D}^{*}$ at $2 \mathrm{~V}$ is closely related to the largest on/off ratio at $1.6 \sim 2.1 \mathrm{~V}$, as shown in Fig. $2 \mathrm{~b}$. The $\mathrm{D}^{*}$ at $2 \mathrm{~V}$ remains high both in the VIS $\left(1.1 \times 10^{11} \mathrm{~cm} \mathrm{~Hz}^{1 / 2} \mathrm{~W}^{-1}\right.$ or Jones at $400 \mathrm{~nm})$ and $\operatorname{NIR}\left(2.4 \times 10^{11}\right.$ Jones at $\left.900 \mathrm{~nm}\right)$ ranges due to its 

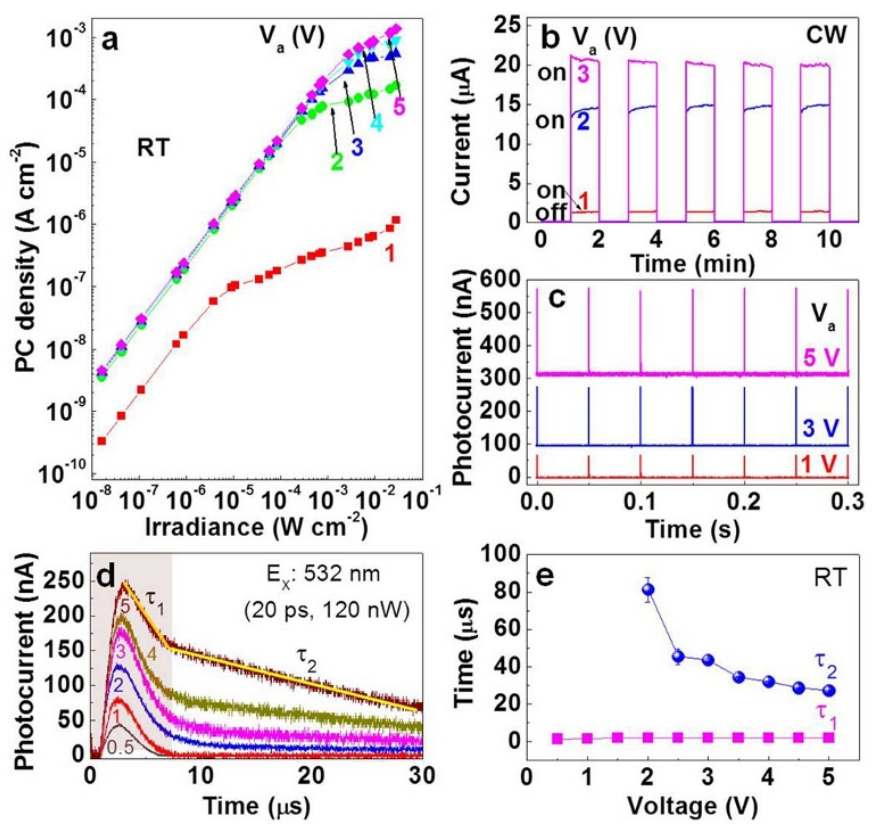

Figure $4 \mid$ Linearity and time response. (a) Irradiance-dependent photocurrent densities at different forward-bias voltages from 1 to 5 V. A 532-nm monochromatic light from a diode laser was used for studying the photosensitivity linearity with light power. Repeated (b) long- and (c) short-term photocurrent switching at various bias voltages by using chopped-continuous-wave- and pulsed-laser lines at $532 \mathrm{~nm}$, respectively. (d) Transient photocurrents at different forward biases from 0.5 to $5 \mathrm{~V}$. The photocurrent switching and transient behaviors were measured by using 20-ps, 20-Hz Nd:YAG laser pulses at $532 \mathrm{~nm}$. (e) The characteristic times $\tau_{1}$ and $\tau_{2}$ as functions of forward bias.

quite-good spectral flatness. These values are comparable to those of compound-semiconductor quantum-dot $\mathrm{PDs}^{16,17}$.

Linearity and time response. Fig. 4a shows $\mathrm{PC}$ density $\left(\mathrm{J}_{\mathrm{PC}}\right)$ vs incident light power (P) of a $532 \mathrm{~nm}$ laser line at various bias voltages, which was obtained at a modulation frequency of $30 \mathrm{~Hz}$. For lower light powers spanning five orders of magnitude, the JPC increases linearly with $\mathrm{P}$ under biases $\geq 2 \mathrm{~V}$, and after a threshold power it deflects from the linear relationship. The threshold power increases with increasing the bias voltage. The $\mathrm{J}_{\mathrm{PC}}$ in the linear region can be expressed by a simple power law: $\mathrm{J}_{\mathrm{PC}} \propto \mathrm{P}^{\alpha}$, where $0.99<\alpha<$ 1.01 in this work. The linear increase of the PC density is attributed to the linear photo-generation of electron-hole pairs. However, at high powers the built-in electric field can be lowered due to a large accumulation of photogenerated carriers at the GQDs/graphene interface states ${ }^{35,36}$, resulting in the saturation of the PC density at high powers.

The linear dynamic range (LDR) or the photosensitivity linearity (typically quoted in $\mathrm{dB})$ is given by $\mathrm{LDR}=20 \log \left(\mathrm{J}^{*}{ }_{\mathrm{PC}} / \mathrm{J}_{\mathrm{d}}\right)$, where $\mathrm{J}^{*}{ }_{\mathrm{PC}}$ is the PC density measured at a light intensity of $1 \mathrm{~mW} / \mathrm{cm}^{2}$ and $\mathrm{J}_{\mathrm{d}}$ is the DC density. The ratio of full-well capacity of today's CMOS image sensors to the number of dark electrons limits dynamic range to about three orders of magnitude in intensity (conventionally expressed as $60 \mathrm{~dB}$ in imaging), in other words, more than $60 \mathrm{~dB}$ is required for the imaging application of PDs. The LDR is $\sim 95 \mathrm{~dB}$ (Supplementary Fig. S14a), which is comparable to that of Si PDs $(\sim 120 \mathrm{~dB})$ and significantly higher than that of InGaAs PDs $(\sim 66 \mathrm{~dB})^{37,38}$. The LDR increases with increasing the bias voltage. The linearity of $\mathrm{R}_{\mathrm{i}}$ with the light power is also enhanced at higher bias voltages (Supplementary Fig. S14b).

Figs. $4 \mathrm{~b}$ and $\mathrm{c}$ show long- and short-term PC switching behaviors at various bias voltages by using chopped-continuous-wave- and pulsed-laser lines at $532 \mathrm{~nm}$, respectively. The lasers were focused onto the PD devices with a spot size of $\sim 5 \times 5 \mathrm{~mm}^{2}$. Within $100 \mu \mathrm{s}$ after the light is turned off the current reaches the initial DC value. Both PC switching behaviors are very regular for every chopped and pulsed light, almost irrespective of the bias voltage. The long-term PC switching responses are almost independent of $\lambda$ (Supplementary Fig. S15).

To probe the charge-transfer dynamics, transient PCs were recorded at much smaller time scale for various bias voltages, as shown in Fig. 4d. The turn-on transient response is characterized by a relatively fast increase in the PC during $\tau_{0}$ ( $\sim$ several tens of $\left.n s\right)$, followed by a relatively slow decay with two characteristic times of $\tau_{1}$ and $\tau_{2}$ to a steady state value at bias voltages $\geq 2 \mathrm{~V}$ (Supplementary Figs. S16a and b). The fast increase is attributed to carriers generated in the region GQDs and swept out as a drift current proportional to the built-in electric field. The rise time is the time required for generation and tunneling in the graphene/GQDs/graphene structures. With increasing the bias voltage, the measured $\tau_{1}$ first increases and then reaches a saturation value of $\sim 2 \mu \mathrm{s}$ at a bias of $2 \mathrm{~V}$ (Supplementary Fig. S16c) whilst the $\tau_{2}$ decreases down to $\sim 30 \mu \mathrm{s}$ at $5 \mathrm{~V}$, as shown in Fig. 4e. The two characteristic times are attributed to the two different current mechanisms of the diffusion $\left(\tau_{1}\right)$ and the drift $\left(\tau_{2}\right)$. As the bias voltage increases the drift velocity of carriers increases, which give rises to the decrease of $\tau_{2}$ at lager bias voltage. However, the current by diffusion does not depend on the bias voltage, but on the incident light power. The electrons and holes photoexcited in GQDs transport to the graphene sheets under a bias while experiencing trappings not only in GQDs but also at the GQDs/ graphene interfaces, which can easily expect $\tau_{1}$ and $\tau_{2}$ to be larger than the decay time in pristine graphene ${ }^{39}$. These behaviors do not seem to be relevant to the RC time constant of the device, measured to be an order of ns (Supplementary Fig. S17b). From the C-V curve, the built-in potential between graphene and GQD is estimated to be $\sim 1.3 \mathrm{~V}$ (Supplementary Fig. S17c), which is very close to the potential barrier $(\sim 1.4 \mathrm{~V})$ obtained from the low-T dark I-V curves (Supplementary Fig. S7), as shown as above, the peak voltage (1.6 $\sim 2.1 \mathrm{~V}$ ) for the largest PC/DC ratio (Fig. 2b), and the threshold voltage $(1 \sim 2 \mathrm{~V})$ for inducing the PC decay with two characteristic times (Fig. 4d).

\section{Discussion}

It should be noted that the measured QEs are the effective ones, i.e., $\eta_{\text {eff }}=\eta G$, where $\eta$ and $G$ are the quantum efficiency and the internal gain of the device, respectively ${ }^{16}$. Extremely-high gain is expected in the GQD PD devices because the gain is given by the ratio of the carrier lifetime $(\tau)$ to the transit time $\left(\tau_{0}\right)$ across the electrodes, $\mathrm{G} \sim \tau / \tau_{0}$, and from the transient PC the lifetime of electron-hole pair is estimated to be several tens of $\mu$ s $\left(\tau \sim\left(\tau_{1}+\tau_{2}\right)\right)$. Within several tens of ns $\left(\sim \tau_{0}\right)$ upon a light pulse, the electrons and holes are produced in GQDs and transit to the graphene sheets. Thus, the current gain in the $\mathrm{PD}$ can reach an order of $10^{3}$, resulting in effective QEs almost over 80\% in the PD devices, as shown in Fig. 3b.

The achievement of the high-performance GQD PDs in this work suggests that GQDs can be employed in various kinds of transparent and foldable optoelectronic devices and will open exciting opportunities not only for the creation of single-function PDs in detecting optical signals in wide spectral range but also for the fabrication of a key component in high-resolution PD arrays for biomedical imaging, remote sensing, optical communication, and OEICs.

\section{Methods}

Preparation of GQDs. GQDs were fabricated by following processes. Graphene oxide (GO) sheets were obtained from natural graphite powder by a modified Hummers method ${ }^{40,41}$. The GO sheets were subsequently deoxidized in a tube furnace at $250^{\circ} \mathrm{C}$ for $2 \mathrm{~h}$ under Ar ambient to prepare reduced graphene oxide powder, $0.05 \mathrm{~g}$ of which were then oxidized in concentrated $10-\mathrm{mL} \mathrm{H}_{2} \mathrm{SO}_{4}$ and $30-\mathrm{mL} \mathrm{HNO}_{3}$ for $20 \mathrm{~h}$ under mild ultrasonication. The mixture was then diluted with $250-\mathrm{mL}$ 
deionized (DI) water and filtered through a $0.02-\mu \mathrm{m}$ nanoporous membrane to remove the acids. The size-reduced/purified 0.2-g GO powder was re-dispersed in 40 $\mathrm{mL}$ DI water and the $\mathrm{pH}$ was tuned to 8 with $\mathrm{NaOH}$. The suspension was transferred to a nitrogen-ambient furnace and heated at $250^{\circ} \mathrm{C}$ for $10 \mathrm{~h}$. After cooling to room temperature, the resulting powder was re-dispersed in $40-\mathrm{mL} \mathrm{DI}$ water for $2 \mathrm{~h}$ under ultrasonication. Then, by filtering the resulting suspension through a $0.2-\mu \mathrm{m}$ nanoporous membrane, a brown filter solution was separated. Since the colloidal solution still contained some large graphene nanoparticles (50-200 nm) emitting weak blue fluorescence, it was further dialyzed in a dialysis bag of $3500 \mathrm{Da}$ molecular weight overnight, thereby producing strongly fluorescent GQDs. The GQDs were separated as different sizes by using 1000, 25,000, and 50,000 Da dialysis bags and a 20-nm nanoporous membrane.

Fabrication of devices. Graphene layers were grown on 70 - $\mu$ m-thick $\mathrm{Cu}$ foils (Wacopa, 99.8 purity) in a graphite-heater-based chemical-vapour-deposition quartz tube furnace at a growth temperature of $1000^{\circ} \mathrm{C}$ with $10-\mathrm{sccm} \mathrm{H}_{2}$ and $20-\mathrm{sccm} \mathrm{CH}_{4}$ flowing at a pressure of 3 Torr. The graphene/Cu stack was spin-coated with poly(methyl methacrylate) (PMMA), and the Cu was then etched in a $1 \mathrm{M}$ ammonium persulfate for $10 \mathrm{~h}$. The graphene/PMMA stack was then placed in deionized water before transferring to the $300 \mathrm{~nm} \mathrm{SiO} /$ p-type Si wafers and blowdried with dry $\mathrm{N}_{2}$. The PMMA/graphene/ $\mathrm{SiO}_{2} / \mathrm{Si}$ stack was then heated on a hot plate in air at $180^{\circ} \mathrm{C}$ for $2 \mathrm{~h}$ to cure the PMMA. After the samples were cooled to room temperature (RT), the PMMA was stripped by soaking them in acetone for $1 \mathrm{~h}$ at RT. Subsequently, the graphene/ $\mathrm{SiO}_{2} / \mathrm{Si}$ stack was put in isopropyl alcohol for $10 \mathrm{~min}$ and dried by blowing $\mathrm{N}_{2}$ to minimize the water traps possibly present at the graphene/ $\mathrm{SiO}_{2}$ interface, and annealed at $400^{\circ} \mathrm{C}$ for $1 \mathrm{~h}$ in vacuum to remove the surface adsobates. A 200- $\mu \mathrm{l}$ solution of GQDs was then dropped and spin-coated on the $10 \times$ $10 \mathrm{~mm}^{2}$ graphene $/ \mathrm{SiO}_{2} / \mathrm{p}$-type $\mathrm{Si}$ wafer, and annealed at $100^{\circ} \mathrm{C}$ for $1 \mathrm{~min}$. This process was 10 times repeated to enhance the density of GQDs. Subsequently, a $5 \times$ $5 \mathrm{~mm}^{2}$ single-layer graphene was transferred on $\sim 1 / 4$ area of the GQDs/graphene/ $\mathrm{SiO}_{2} / \mathrm{p}$-type $\mathrm{Si}$ wafer, and annealed at $400^{\circ} \mathrm{C}$ for $1 \mathrm{~h}$ in vacuum. As a result, the graphene/GQDs/graphene sandwich structure was formed on the $\sim 1 / 4$ area of the $\mathrm{SiO}_{2} / \mathrm{p}$-type $\mathrm{Si}$ wafer. Ag or Au electrodes of $1 \mathrm{~mm}$ diameter and $1 \mu \mathrm{m}$ thickness were deposited on the top of both graphene sheets by RF sputtering to complete the GQD $\mathrm{PD}$ device. The junction between $\mathrm{Ag}$ (or $\mathrm{Au}$ ) and graphene was demonstrated to be ohmic in our previous report ${ }^{42}$. For the graphene field effect transistors, $\mathrm{Cr} / \mathrm{Au}$ source and drain electrodes of $30 / 10 \mu \mathrm{m}$ in width/length were fabricated on top of graphene via the processes including reactive ion etching, metal deposition, and lift-off, similar to those in the previous publications ${ }^{43,44}$.

Measurements. PL spectra were measured at room temperature using the $325 \mathrm{~nm}$ line of a He laser as the excitation source. Emitted light was collected by a lens and analyzed using a grating monochromator and a GaAs photomultiplier (PM) tube. Standard lock-in detection techniques were used to maximize the signal-to-noise ratio. The topographic image and height profile of graphene were obtained in a noncontact mode of atomic force microscope (Park system, model XE-100). Raman spectroscopy with an excitation wavelength of $532 \mathrm{~nm}$ was used to characterize the optical properties of graphene films. The transmittance of the sample was measured by UV-VIS-NIR optical spectrometer (Agilent Model Cary 5E). I-V measurements to characterize the electrical behaviors of GQD PDs were carried out using a Keithley 2400 source meter controlled by a LabView program. The dark current noise under forward bias was measured with a dynamic signal analyzer (Agilent 35670A) coupled to a low-noise current preamplifier (Stanford Research SR570) in the frequency range of $1 \mathrm{~Hz}$ to $10 \mathrm{kHz}$. Biases, when needed, were supplied by the current preamplifier. During the measurements, the PDs were mounted in a dark, electrically-shielded, and optically-sealed chamber on the optical table to reduce vibrational noise. The spectral photoresponse was detected by focusing the light onto the PD devices with a spot size of $\sim 5 \times 5 \mathrm{~mm}^{2}$ in the $300-1100 \mathrm{~nm}$ wavelength range. For this purpose, we used a $450 \mathrm{~W}$ xenon light source and a 0.25 -m grating monochromator (Newport Cornerstone 260 1/4 m monochromator). The incident light intensity was monitored by a motorized variable wheel attenuator and a Newport calibrated UV enhanced silicon photodiode. The power density of the incident light on the sample surface was about $1 \sim 320 \mu \mathrm{W} / \mathrm{cm}^{2}$ depending on the wavelength in the range of $300 \sim 1100 \mathrm{~nm}$. A 532-nm monochromatic light from a diode laser was used for studying the photosensitivity linearity with light power. The light was modulated with a mechanical chopper (Standard Research Systems) with a frequency of $30 \mathrm{~Hz}$ and the PC response at various bias voltages was recorded with a Signal Recovery 7265 DSP lock-in amplifier. Optical neutral density filters were used to vary the light intensity from $\mathrm{nW}$ up to $7.2 \mathrm{~mW}$. (maximum power: $90 \mathrm{~mW}$ ). Transient photocurrent studies were conducted using laser pulses from the Nd:YAG laser. (532-nm wavelength, 20 ps duration, and $20-\mathrm{Hz}$ repetition rate, generated by a Continuum Leopard-D20 Nd:YAG laser), which were focused onto the PD devices with a spot size of $\sim 5 \times$ $5 \mathrm{~mm}^{2}$. A laser power meter (Laser probe, $\mathrm{Rj}-760$ ) was used to measure the average power of the laser pulses. The laser pulse temporal profiles and transient PC were monitored with the 50 -ohm-terminated, $500-\mathrm{MHz}$-bandwidth digital oscilloscope (Tektronix DPO 4054). All measurements were performed in a dark, shielded enclosure under vacuum.

1. Han, M. Y., Özyilmaz, B., Zhang, Y. \& Kim, P. Energy band-gap engineering of graphene nanoribbons. Phys. Rev. Lett. 98, 206805 (2007).
2. Kosynkin, D. V. et al. Longitudinal unzipping of carbon nanotubes to form graphene nanoribbons. Nature 458, 872-876 (2009).

3. Yan, X., Cui, X. \& Li, L.-S. Synthesis of Large, stable colloidal graphene quantum dots with tunable size. J. Am. Chem. Soc. 132, 5944-5945 (2010).

4. Pan, D., Zhang, J., Li, Z. \& Wu, M. Hydrothermal route for cutting graphene sheets into blue-luminescent graphene quantum dots. Adv. Mater. 22, 734 (2010).

5. Mueller, M. L., Yan, X., McGuire, J. A. \& Li, L.-S. Triplet states and electronic relaxation in photoexcited graphene quantum dots. Nano Lett. 10, 2679-2682 (2010).

6. Lu, J., Yeo, P. S. E., Gan, C. K., Wu, P. \& Loh, K. P. Transforming $\mathrm{C}_{60}$ molecules into graphene quantum dots. Nature Nanotech. 6, 247-252 (2011).

7. Ponomarenko, L. A. et al. Chaotic Dirac billiard in graphene quantum dots. Science 320, 356-358 (2008).

8. Geim, A. K. \& Novoselov, K. S. The rise of graphene. Nature Mater. 6, 183-191 (2007).

9. Geim, A. K. Status and prospects. Science 324, 1530-1534 (2009).

10. Ritter, K. A. \& Lyding, J. W. The influence of edge structure on the electronic properties of graphene quantum dots and nanoribbons. Nature Mater. 8, 235-242 (2009).

11. Güçlü, A. D., Potasz, P. \& Hawrylak, P. Excitonic absorption in gate-controlled graphene quantum dots. Phys. Rev. B 82, 155445 (2010).

12. Kim, S. et al. Anomalous behaviors of visible luminescence from graphene quantum dots: interplay between size and shape. ACS Nano 6, 8203 (2012).

13. Brass, E., Hilleringmann, U. \& Schumacher, K. System integration of optical devices and analog CMOS amplifiers. IEEE J. Solid-State Circuits 5, 1006-1010 (1994).

14. Garrett, L. D., Qi, J., Schow, C. L. \& Campbell, J. C. A silicon-based integrated NMOS-p-i-n photoreceiver. IEEE Trans. Electron Dev. 43, 411-416 (1996).

15. "European commission technology roadmap-optoelectronics interconnects for integrated circuits", Office for Official Publications of the European Communicaties, Luxemburg, 1998.

16. Konstantatos, G. \& Sargent, E. H. Nanostructured materials for photon detection Nature Nanotech. 5, 391-400 (2010)

17. Lee, J.-S., Kovalenko, M. V., Huang, J., Chung, D. S. \& Talapin, D. V. Band-like transport, high electron mobility and high photoconductivity in all-inorganic nanocrystal arrays. Nature Nanotech. 6, 348 (2011).

18. Lee, E. J. H., Balasubramanian, K., Weitz, R. T., Burghard, M. \& Kern, K. Contact and edge effects in graphene devices. Nature Nanotech. 3, 486 (2008).

19. Xu, X., Gabor, N. M., Alden, J. S., van der Zande, A. M. \& McEuen, P. L. Photothermoelectric effect at a graphene interface junction. Nano Lett. 10, 562-566 (2010).

20. Xia, F., Mueller, T., Lin, Y.-M., Valdes-Garcia, A. \& Avouris, P. Ultrafast graphene photodetector. Nature Nanotech. 4, 839-843 (2009).

21. Mueller, T., Xia, F. \& Avouris, P. Graphene photodetectors for high-speed optical communications. Nature Photon. 4, 297-301 (2010).

22. Park, J., Ahn, Y. H. \& Ruiz-Vargas, C. Imaging of photocurrent generation and collection in single-layer graphene. Nano Lett. 9, 1742 (2009).

23. Britnell, L. et al. Strong Light-Matter Interactions in Heterostructures of Atomically Thin Films. Science 340, 1311-1314 (2013).

24. Esaki, L. Discovery of the Tunnel Diode. IEEE Trans. Electron Dev. 23, 644-647 (1976).

25. Kang, Y.-J., Kang, J. \& Chang, K. J. Electronic structure of graphene and doping effect on $\mathrm{SiO}_{2}$. Phys. Rev. B 78, 115404 (2008)

26. Xu, H., Chen, Y., Zhang, J. \& Zhang, H. Investigating the mechanism of hysteresis effect in graphene electrical field device fabricated on $\mathrm{SiO}_{2}$ substrates using Raman spectroscopy. Small 8, 2833-2840 (2012).

27. Hwang, E., Hu, B. Y.-K. \& Das Sarma, S. Inelastic carrier lifetime in graphene. Phys. Rev. B 76, 115434 (2007).

28. Kim, R., Perebeinos, V. \& Avouris, P. Relaxation of optically excited carriers in graphene. Phys. Rev. B 84, 075449 (2011).

29. Winzer, T. \& Malić, E. Impact of Auger processes on carrier dynamics in graphene. Phys. Rev. B 85, 241404 (2012).

30. Winzer, T., Knorr, A. \& Malić, E. Carrier multiplication in graphene. Nano Lett. 10, 4839-4843 (2010)

31. Song, J. C. W., Rudner, M. S., Marcus, C. M. \& Levitov, L. S. Hot carrier transport and photocurrent response in graphene. Nano Lett. 11, 4688-4692 (2011).

32. Yu, Z., Aceves-Mijares, M., Lopez, J. A. L. \& Deng, J. Nanocrystalline Si-based metal-oxide-semiconductor photodetectors. Proc. of SPIE 7381, 73811H (2009)

33. Shaw, P.-S., Larason, T. C., Gupta, R., Brown, S. W. \& Lykke, K. R. Improved nearinfrared spectral responsivity Scale. J. Res. Natl. Inst. Stand. Technol. 105, 689-700 (2000).

34. Pal, B. N. et al. High-Sensitivity p-n Junction Photodiodes Based on PbS Nanocrystal Quantum Dots. Adv. Funct. Mat. 22, 1741-1748 (2012).

35. Konstantatos, G. et al. Hybrid graphene-quantum dot phototransistors with ultrahigh gain. Nature Nanotech. 7, 363-368 (2012).

36. Roy, K. et al. Graphene- $\mathrm{MoS}_{2}$ hybrid structures for multifunctional photoresponsive memory devices. Nature Nanotech. 8, 826-830 (2013)

37. Manga, K. K. et al. High-performance broadband photodetector using solution processible $\mathrm{PbSe}-\mathrm{TiO}_{2}$-graphene hybrids. Adv. Mater. 24, 1697-1702 (2012).

38. Gong, X. et al. High-detectivity polymer photodetectors with spectral response from $300 \mathrm{~nm}$ to $1450 \mathrm{~nm}$. Science 325, 1665-1667 (2009). 
39. Sun, D. et al. Ultrafast hot-carrier-dominated photocurrent in graphene. Nature Nanotech. 7, 114-118 (2012).

40. Xu, Y., Bai, H., Lu, G., Li, C. \& Shi, G. Flexible Graphene Films via the Filtration of Water-Soluble Noncovalent Functionalized Graphene Sheets. J. Am. Chem. Soc. 130, 5856-5857 (2008)

41. Pan, D. et al. Li Storage Properties of Disordered Graphene Nanosheets. Chem. Mater. 21, 3136-3142 (2009).

42. Kim, S. et al. Graphene p-n vertical tunneling diodes. ACS Nano 7, 5168-5174 (2013).

43. Das, A. et al. Monitoring Dopants by Raman Scattering in an Electrochemically Top-Gated Graphene Transistor. Nat. Nanotechnol. 3, 210-215 (2008).

44. Pisana, S. et al. Breakdown of the adiabatic Born-Oppenheimer approximation in graphene. Nat. Materials 6, 198-201 (2007).

\section{Acknowledgments}

This work was supported by Basic Science Research Program through the National Research Foundation of Korea (NRF) funded by the Ministry of Science, ICT \& Future Planning (No. 2014022732 and NRF-2014R1A1A1A05008463).

\section{Author contributions}

J.H.K. and C.W.J. deposited graphene sheets by chemical vapour deposition. S.W.H. prepared GQDs with S.S.K. J.M.K. structurally characterized GQDs. K.W.L. carried out optical measurements. S.K. fabricated GQD PDs with D.H.S. C.O.K. characterized performances of GQD PDs. E.H. contributed to the theoretical interpretation of the results. S.-H.C. initiated, supervised the work, and wrote the paper. All authors discussed the results and commented on the manuscript.

\section{Additional information}

Supplementary information accompanies this paper at http://www.nature.com/ scientificreports

Competing financial interests: S.-H.C., S.K., C.O.K. and D.H.S. have a Korea patent pending (10-2013-0053894) on the GQD PD production technology, reported in this paper. How to cite this article: Kim, C.O. et al. High-performance graphene-quantum-dot photodetectors. Sci. Rep. 4, 5603; DOI:10.1038/srep05603 (2014).

This work is licensed under a Creative Commons Attribution-NonCommercialNoDerivs 4.0 International License. The images or other third party material in this article are included in the article's Creative Commons license, unless indicated otherwise in the credit line; if the material is not included under the Creative Commons license, users will need to obtain permission from the license holder in order to reproduce the material. To view a copy of this license, visit http:// creativecommons.org/licenses/by-nc-nd/4.0/ 